\title{
ILUSTRAÇÃO DE CARACTERES MICROSCÓPICOS DE DROGAS VEGETAIS PARA O CONTROLE DE QUALIDADE FARMACOGNÓSTICO \\ I. PEUMUS BOLDUS MOLINA E MIKANIA GLOMERATA SPRENG.
}

\author{
ILLUSTRATION OF VEGETAL DRUG MICROSCOPIC \\ CHARACTERS FOR THE PHARMACOGNOSTIC QUALITY \\ CONTROL
}

I. Peumus boldus MOLINA E Mikania glomerata SPRENG.

DUARTE, M. do R.

Laboratório de Farmacognosia, Departamento de Farmácia, Universidade Federal do Paraná (UFPR), Av. Pref. Lothário Meissner, 632, Jardim Botânico, 80210-170, Curitiba, PR, marciard@ufpr.br

RECEBIDO: JUN/07 ACEITE: SET/07

\begin{abstract}
RESUMO
Nas monografias farmacopéicas de drogas vegetais constam diversas análises a serem realizadas para o controle de qualidade farmacognóstico. A etapa inicial se refere à identificação da amostra, com base em descrições macro e microscópica do material. Como forma de complementar essas descrições e favorecer o reconhecimento dos caracteres morfológicos, este trabalho objetivou apresentar ilustrações anatômicas de duas drogas farmacopéicas: Peumus boldus Molina e Mikania glomerata Spreng. Amostras de folhas foram fixadas, seccionadas à mão livre ou emblocadas em glicometacrilato e seccionadas em micrótomo, e os cortes foram corados. Dentre os caracteres anatômicos observados, as ilustrações de $P$. boldus destacam os tricomas tectores estelares, a hipoderme junto à face adaxial e as células oleíferas no mesofilo dorsiventral. Em M. glomerata, os tricomas glandulares curvos inseridos em depressão epidérmica, a hipoderme descontínua e os dutos secretores nas proximidades dos feixes vasculares são indicados nas ilustrações.
\end{abstract}

Palavras-chave: droga vegetal; farmacopéia; ilustração; microscopia.

\begin{abstract}
The vegetal drug monographs from pharmacopoeias present several analyses to be carried out for the pharmacognostic quality control. The first essay is related to the identification of the drug, by means of macro and microscopic descriptions. Aiming to supply additional information and to favour the recognition of morphological characters, this work has presented anatomical illustration of two vegetal drugs: Peumus boldus Molina e Mikania glomerata Spreng. Foliar samples were fixed, either sectioned by freehand or embedded in glycol methacrylate and sectioned by microtome, and stained. Among the anatomical features observed, the illustrations of $P$. boldus depict stellate non-glandular trichomes, the hypodermis next to the adaxial surface and secretory cells in the dorsiventral mesophyll. In M. glomerata, the glandular trichomes, twisted and located in an epidermal depression, the discontinuous hypodermis and secretory ducts near the vascular bundles are shown in the illustrations.
\end{abstract}

Key words: Illustration; microscopy; pharmacopoeia; vegetal drug. 


\section{INTRODUÇÃO}

As Farmacopéias incluem monografias de diversas drogas vegetais, nas quais são descritas as diferentes análises a serem conduzidas para o controle de qualidade farmacognóstico. A etapa inicial se refere à caracterização morfológica da droga vegetal, que compreende a identificação macro e microscópica do material. Essa investigação confirma a identidade da droga e antecede as pesquisas de princípios ativos e/ou marcadores químicos, e os ensaios de impureza e falsificação.

Apenas recentemente publicaram-se algumas monografias com a descrição morfológica detalhada acompanhada de ilustrações, como forma de facilitar o reconhecimento das estruturas vegetais. Dessa maneira, a título de exemplificação, as monografias de capim-limão, espinheira-santa, carqueja e guaraná na F. Bras. IV (FARMACOPÉIA, 1988-2003) contam com figuras para representar os caracteres morfológicos próprios. Esse recurso complementar favorece de tal maneira a confirmação da identidade da droga, na medida em que as imagens traduzem a nomenclatura botânica que poucos dominam, que se cogitou a possibilidade da elaboração de ilustrações para as demais monografias.

Por essa razão, este trabalho objetivou registrar por meio de fotografias os caracteres anatômicos de drogas farmacopéicas, fornecendo um recurso adicional para facilitar a análise morfológica conduzida por profissionais que realizam o controle de qualidade, principalmente em indústrias de fitoterápicos.

As drogas selecionadas para serem ilustradas neste trabalho foram o boldo-do-chile e o guaco. A primeira é definida como sendo constituída de folhas de Peumus boldus Molina (Monimiaceae) (FARMACOPÉIA, 1988-2003) e apresenta alcalóides isoquinólicos, dentre os quais se encontra a boldina, bem como óleo essencial e flavonóides (FELTROW; AVILA, 2000; NEWALL et al., 2002; SKIDMORE-ROTH, 2004). O boldo-do-chile possui reconhecidamente atividades estomáquica, diurética e colagoga (NEWALL et al., 2002). O guaco consiste de folhas de Mikania glomerata Spreng. (Asteraceae) (SILVA, 1926), nas quais foram detectados principalmente cumarinas e óleo essencial. Na terapêutica, o guaco é empregado como broncodilatador e expectorante (LORENZI; MATOS, 2002).

\section{MATERIAL E MÉTODOS}

O material botânico foi coletado e identificado no Departamento de Botânica, do Setor de Ciências Biológicas, da Universidade Federal do Paraná, em meados de 2002.

Amostras foram fixadas em FAA 70 (JOHANSEN, 1940) e posteriormente armazenadas em etanol a $70 \%$ (BERLYN; MIKSCHE, 1976). Para a montagem de lâminas semipermanentes, o material foi seccionado no sentido transversal à mão livre e corado com azul de astra e fucsina básica (ROESER, 1972).

Paralelamente, lâminas permanentes foram confeccionadas, empregando-se a técnica de inclusão em glicol metacrilato (FEDER; O'BRIEN, 1968) e seccionamento transversal em micrótomo rotatório. Os cortes obtidos foram corados com a dupla coloração azul de astra e fucsina básica modificada por BRITO e ALQUINI (1996).

Fotomicrografias em papel foram feitas por meio de câmera acoplada ao microscópio óptico Olympus BX40 equipado com a unidade de controle PM20. As fotos coloridas, com as respectivas escalas micrométricas, foram analisadas, digitalizadas e transformadas em tons de cinza.

Para a elaboração das ilustrações, a indicação dos caracteres anatômicos foi feita com base nas descrições microscópicas adaptadas das monografias do boldo-do-chile da F. Bras. IV (FARMACOPÉIA,1988-2003) e do guaco da F. Bras. I (SILVA, 1926).

\section{RESULTADOS}

\section{Boldo-do-chile}

A folha de $P$. boldus apresenta nervura mediana côncavo-convexa em secção transversal (Fig. 1A), mostrando um feixe vascular colateral em arco aberto, envolto por uma bainha contínua de fibras esclerenquimáticas (Fig. 1A) ou por fibras sob a forma de ilhotas, na face abaxial. Nas extremidades do conjunto descrito, voltados para a face adaxial, podem ser encontrados feixes vasculares acessórios.

No limbo, a epiderme na superfície adaxial é formada por células de contorno poligonal, quando vista frontalmente, de paredes quase retas e recobertas por cutícula lisa e espessa. Essa face não possui estômatos, 
mas apresenta pequenas protuberâncias pluricelulares, onde se acham inseridos tricomas tectores simples, bifurcados ou estrelados (estelares) (Fig. 1D, 1E), freqüentemente caducos. Segue-se uma hipoderme (Fig. 1A-1C), formada por uma ou raramente duas camadas de células parenquimáticas, relativamente maiores, achatadas, incolores e de paredes espessadas. O mesofilo consiste de uma ou duas camadas de parênquima paliçádico e vários estratos de parênquima esponjoso (Fig. 1A, 1B). Em meio ao clorênquima, são encontradas células oleíferas (Fig. 1A-1C), volumosas, globosas e de paredes suberizadas. Também, podem ser encontrados pequenos cristais aciculares.

A epiderme na superfície abaxial apresenta numerosos estômatos (Fig. 1A, 1B) anomocíticos, rodeados de até sete células adjacentes. Em vista frontal, as células da face abaxial têm paredes mais onduladas do que as células da face oposta e apresentam menor número de tricomas, geralmente estrelados e caducos.

\section{Guaco}

Na lâmina foliar de M. glomerata, em vista frontal, a epiderme apresenta células poligonais de paredes levemente curvas na face adaxial e de paredes ondeadas na superfície abaxial. Esta possui estômatos (Fig. $2 A$ ), circundados por três a quatro células não diferenciadas (anomocíticos). Ambas as faces possuem tricomas glandulares pluricelulares (curvos) (Fig. 2A, 2B), do tipo geral das Asteraceae, mais freqüentes na face abaxial e situados em depressões epidérmicas.

O mesofilo é heterogêneo assimétrico (dorsiventral) (Fig. 2A), formado na parte superior por uma ou duas camadas de células paliçádicas e na inferior por um parênquima lacunoso (esponjoso), que compreende células arredondadas ou elípticas. Subjacente à face adaxial, ocorre uma camada parenquimática descontínua que constitui a hipoderme (Fig. 2A).

A nervura mediana, nitidamente biconvexa em secção transversal, apresenta de três a cinco feixes vasculares ovais (Fig. 2C), dispostos em arco, do tipo colateral, com calotas esclerenquimáticas apostas ao xilema e ao floema. Nas proximidades dos feixes, notam-se pequenos canais (dutos) secretores (Fig. 2C, 2D).

\section{DISCUSSÃO}

Os caracteres anatômicos foliares de $P$. boldus são coerentes com a família Monimiaceae (METCALFE; CHALK, 1950; 1988) e alguns deles merecem destaque pelo fato de favorecerem a identificação microscópica da espécie. Desse modo, a ocorrência de estômatos anomocíticos exclusivamente na face abaxial, tricomas tectores estelares, hipoderme junto à superfície adaxial e células oleíferas no mesofilo (METCALFE; CHALK, 1950; 1988; CRONQUIST, 1981) constituem-se em estruturas ilustradas neste trabalho e que são facilmente reconhecíveis no boldo-do-chile. Mesmo no material dessecado, que representa a droga vegetal, esses caracteres podem ser visualizados, provavelmente em razão da textura coriácea da folha.

Com relação a $M$. glomerata, os aspectos foliares observados têm correspondência com as Asteraceae (METCALFE; CHALK, 1950; 1988), podendo-se destacar a hipoderme, os dutos secretores e os tricomas glandulares. Estes assumem um formato caracteristicamente curvo, que é típico de Mikania (RODRIGUES et al., 1996). Neste estudo, as ilustrações revelam esses aspectos, no entanto, os mesmos podem não ser prontamente distinguíveis. A presença de tricomas glandulares é relevante, porém a peculiar disposição em curvatura desse anexo epidérmico faz com que freqüentemente seja destacado da epiderme por ocasião do seccionamento do material, dificultando a sua localização. Adicionalmente, a hipoderme, pelo fato de ser descontínua, nem sempre é identificada com facilidade. Diante dessas constatações, a ilustração dos caracteres anatômicos do material analisado favorece a identificação da droga vegetal, particularmente para o profissional menos experiente. 


\section{REFERÊNCIAS}

BERLYN, G. P.; MIKSCHE, J. P. Botanical microtechnique and cytochemistry. Ames: lowa State University Press, 1976.

BRITO, J. F. A.; ALQUINI, Y. A new method for staining botanical material embedded in glycol methacrylate (GMA). Braz. Arch. Biol. Technol., Curitiba, v. 39, p. 949-51, 1996.

CRONQUIST, A. An integrated system of classification of flowering plants. New York: Columbia University Press, 1981. FARMACOPÉIA Brasileira. 4 ed. São Paulo: Atheneu, 1988-2003.

FEDER, N.; O'BRIEN, T. P. Plant microtechnique: some principles and new methods. Am. J. Bot., St. Louis, v. 55, p. 123-42, 1968.

FELTROW, C. W.; AVILA, J. R. Manual de medicina alternativa. Rio de Janeiro: Guanabara Koogan, 2000.

JOHANSEN, D. A. Plant microtechnique. New York: McGraw-Hill Book, 1940.

LORENZI, H.; MATOS, F. J. A. Plantas medicinais do Brasil: nativas e exóticas. Nova Odessa: Plantarum, 2002.

METCALFE, C. R.; CHALK, L. Anatomy of the dicotyledons. Oxford: Clarendon, 1988.

METCALFE, C. R.; CHALK, L. Anatomy of the dicotyledons: leaves, stem, and wood in relation to taxonomy, with notes on economic uses. Oxford: Clarendon Press, 1950.

NEWALL, C. A.; ANDERSON, L. A.; PHILLIPSON, J. D. Plantas medicinais. São Paulo: Premier, 2002.

RODRIGUES, R. F. O.; OLIVEIRA, F.; KATO, E. T. M. Morfodiagnose da droga conhecida como cipó-almécega - Mikania malacolepsis Robinson. Rev. Farm. Bioquim. USP, São Paulo, v. 32, p. 37-44, 1996.

ROESER, K. R. Die Nadel der Schwarzkiefer-Massenprodukt und Kunstwerk der Natur. Mikrokosmos, Stuttgart, v. 61, p. 33-6, 1972.

SILVA, R. A. D. Pharmacopéia dos Estados Unidos do Brasil. São Paulo: Nacional, 1926.

SKIDMORE-ROTH, L. Handbook of herbs and natural supplements. 2nd. ed. St. Louis: Mosby, 2004. 


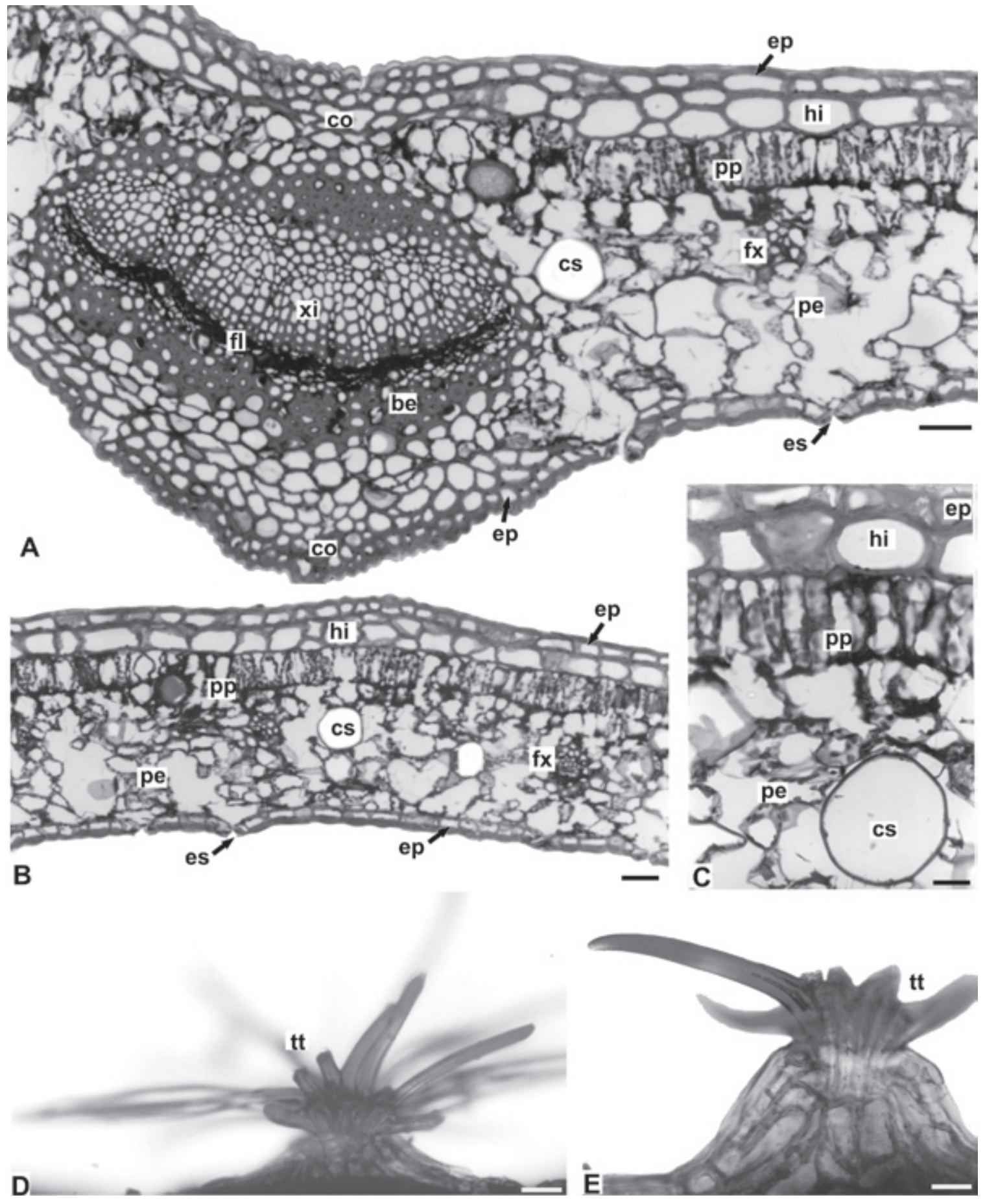

FIGURAS 1A-E. BOLDO-DO-CHILE (PEUMUS BOLDUS MOLINA, MONIMIACEAE) - SECÇÃO TRANSVERSAL DA FOLHA: A. NERVURA MEDIANA E PARTE DA REGIÃO INTERNERVURAL; B. REGIÃO INTERNERVURAL, MOSTRANDO O MESOFILO DORSIVENTRAL; C. DETALHE DA HIPODERME E DE UMA CÉLULA OLEÍFERA; D, E. PORMENOR DE TRICOMAS TECTORES ESTELARES. BE - BAINHA ESCLERENQUIMÁTICA, CO COLÊNQUIMA, CS - CÉLULA SECRETORA, EP - EPIDERME, ES - ESTÔMATO, FL - FLOEMA, FX - FEIXE VASCULAR, HI - HIPODERME, PE - PARÊNQUIMA ESPONJOSO, PP - PARÊNQUIMA PALIÇÁDICO, TT TRICOMA TECTOR ESTELAR, XI - XILEMA. BARRA = $50 \mu \mathrm{m}(\mathrm{A}, \mathrm{B}), 20 \mu \mathrm{m}(\mathrm{C}-\mathrm{E})$ 

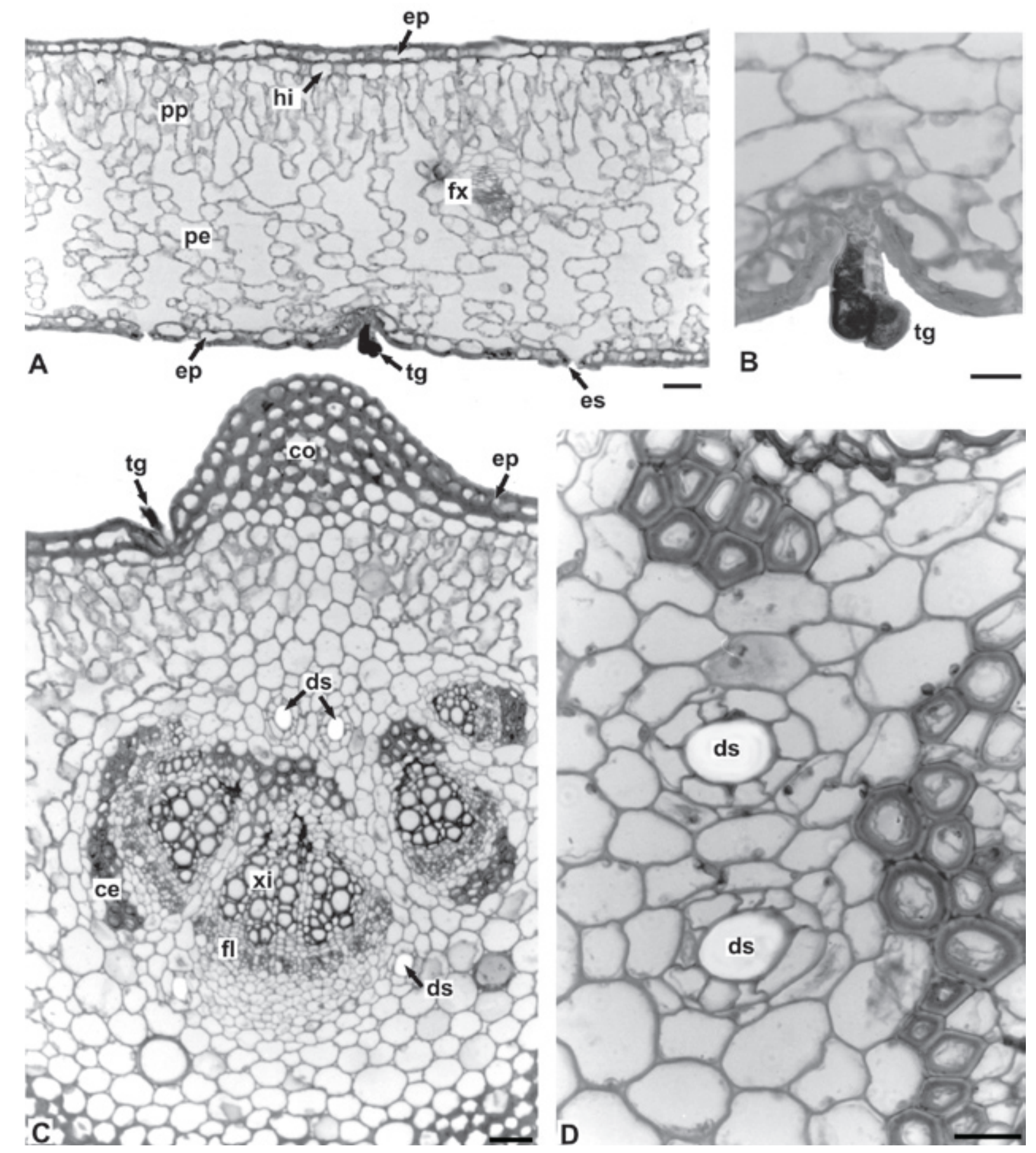

FIGURAS 2A-D. GUACO (MIKANIA GLOMERA SPRENG., ASTERACEAE) - SECÇÃO TRANSVERSAL DA FOLHA: A. REGIÃO INTERNERVURAL, EM QUE SE NOTA O MESOFILO DORSIVENTRAL; B. DETALHE DE UM TRICOMA GLANDULAR CURVO; C. FEIXES VASCULARES NA NERVURA MEDIANA; D. DUTOS SECRETORES NAS PROXIMIDADES DO SISTEMA VASCULAR. CE - CALOTA ESCLERENQUIMÁTICA, CO - COLÊNQUIMA, DS - DUTO SECRETOR, EP - EPIDERME, ES - ESTÔMATO, FL - FLOEMA, FX - FEIXE VASCULAR, HI - HIPODERME, PE - PARÊNQUIMA ESPONJOSO, PP - PARÊNQUIMA PALIÇÁDICO, TG - TRICOMA GLANDULAR CURVO, XI - XILEMA. BARRA $=50 \mu \mathrm{m}(\mathrm{A}, \mathrm{C}), 20 \mu \mathrm{m}(\mathrm{B}, \mathrm{D})$ 